\title{
Does Hysteroscopy in Women with Persistent Gestational Trophoblastic Disease Reduce the Need for Chemotherapy? A Prospective, Single Arm, Clinical Trial Pilot Study
}

Fatemeh Davari Tanha

Tehran University of Medical Sciences

\section{Saghar Samimi Sede}

Tehran University of Medical Sciences

Fariba Yarandi

Tehran University of Medical Sciences

Elham Shirali

Tehran University of Medical Sciences

Malihe Fakehi

Iran University of Medical Sciences

\section{Zeinab Shaker}

Tehran University of Medical Sciences

Mahsa Ghajarzadeh

Tehran University of Medical Sciences

marjan ghaemi ( $\triangle$ MARJAN_GHAEMI@YAHOO.COM )

Tehran University of Medical Sciences

\section{Original Article}

Keywords: Hysteroscopy, Persistent Gestational Trophoblastic Disease, Molar Pregnancy, Beta-hCG, Chemotherapy, Hydatidiform mole

Posted Date: August 25th, 2020

DOI: https://doi.org/10.21203/rs.3.rs-63085/v1

License: (9) (1) This work is licensed under a Creative Commons Attribution 4.0 International License. Read Full License 
Version of Record: A version of this preprint was published at Gynecological Surgery on June 3rd, 2021. See the published version at https://doi.org/10.1186/s10397-021-01094-7. 


\section{Abstract}

Background: This study aimed to describe the efficacy of hysteroscopy in the management of women with persistent gestational trophoblastic disease to decrease the chemotherapy need.

Materials and methods: This prospective, single-arm, clinical trial study was conducted at a tertiary educational referral hospital between September 2018 and September 2019. In the follow-up of the patients diagnosed with hydatidiform mole and managed by uterine evacuation, 30 cases developed low risk persistent gestational trophoblastic disease. Hysteroscopy was performed for removal of persisted trophoblastic tissue, before chemotherapy. Serum beta-hCG titer was measured before and 7 days after the procedure.

Results: Mean $\pm S D$ age of the participants was $31.4 \pm 4.6$ years. There was a significantly difference $(p=0.06)$ between that mean \pm SD of beta-hCG titer before $(8168.4 \pm 1758)$ and after $(2648.8 \pm 5888)$ treatment. Two (6.6\%) cases needed chemotherapy due to no decrease in beta-hCG titer.

Conclusion: Although, hysteroscopy may play a significant role in the management of persistent gestational trophoblastic disease but requires validation in larger prospective randomized studies and longer follow-up.

\section{Introduction}

Hydatidiform mole (HM) also known as molar pregnancy occurs by placental pathology of androgenetic origin (1). This phenomenon happens with abnormal hyperproliferation of the placental villi and hydropic degeneration (2). HM belongs to the gestational trophoblastic diseases (GTD) as the most common pathologies and could locally invade the uterus and metastasize potentially (1). HM is a premalignant condition and categorized as complete and partial and may develop into a malignancy. Malignant disease is referred to as gestational trophoblastic neoplasia (GTN) (3).

The incidence of HM varies from 66 to 121 per 100,000 pregnancies in North America, and Europe whereas Asia, Latin America, and Middle East report a higher rate (23 to 1299 per 100,000 pregnancies) (4). Two well-known risk factors of $\mathrm{HM}$ are maternal age and previous hydatidiform mole (5) as the chance of repeating is $10-20$ folds more than the general population (6).

The common features are vaginal bleeding, uterine enlargement, pelvic pain, hyperemesis as well as hyperthyroidism, pregnancy-induced hypertension, and ovarian theca lutein cyst as less or late features (7). History taking, physical examination, elevated beta-hCG, chest radiograph, and ultrasound findings are routine diagnostic evaluation; while computed tomography (CT) scan and magnetic resonance imaging (MRI) may also been employed (8). Surgical removal (Uterine evacuation or hysterectomy) is the central component of HM treatment (9). 
Fifteen percent of complete hydatidiform moles will have malignant transformation to gestational trophoblastic neoplasia while this rate could be $0.5-1 \%$ in partial HM (10); consequently, they need chemotherapy despite its complications and disadvantages.

Persistent positive beta-hCG titer helps us to detect development of GTN in the follow-up period and further management is mandatory (11). In the cases of remained positive beta-hCG titer, further intervention by the second curettage for removing the residual trophoblastic tissue may develop intrauterine adhesions which causes infertility (12). Second curettage was accepted as an alternative to chemotherapy for non-metastatic and low-grade GTN for patients who have the intrauterine disease, although, it might not reduce the need for chemotherapy in persisted cases (13).

Hysteroscopy resection was applied for removing of residual of trophoblastic tissue. Considering that hysteroscopy has fewer complications than curettage (12), we designed this study to assess whether hysteroscopy in persisted gestational trophoblastic diseases (PGTD) could reduce the need for further chemotherapy.

\section{Materials And Methods}

\section{Study Overview}

This prospective, single-arm, clinical trial study was conducted at a tertiary educational referral hospital affiliate to Tehran University of Medical Sciences between September 2018 and September 2019. All participants suspected with hydatidiform were recruited in the study. Lab data such as kidney, liver and thyroid function test, complete blood count (CBC) and electrolytes was obtained. Indeed, beta-hCG titer was measured as baseline. An abdomino-pelvic ultrasound performed to find any evidence of an invasive mole, exclude a coexisting pregnancy, and look for possible metastatic disease. Chest radiography was ordered for all patients.

In the participants who wanted to preserve fertility, uterine evacuation performed. After dilatation, the molar tissue was evacuated with suction cannula 9 gauge and sharp curettage by curette at the end of the procedure.

International Federation of Gynecologists and Obstetricians (FIGO) standardized created criteria to follow the gestational trophoblastic disease (14):

1. Beta- $h$ CG plateau four values $\pm 10 \%$ recorded over a 3-week duration.

2. Elevated beta- hCG level more than $10 \%$ of three values recorded over a 2 -week duration

3. Detectable beta-hCG for more than 6 months after evacuation.

In the follow-up period, patients were visited every week and the beta-hCG was measured by cobas analyzer for free beta subunit by ECL (Electrochemiluminescence). In persisted PGD according to the mentioned criteria, hysteroscopy was performed by surgeon with 15 years' experience in the field of 
hysteroscopy, with $26 \mathrm{~F}$ Karl Storz bipolar resectoscope fitted with an $8 \mathrm{~mm}$ cutting loop. Saline was used to distend the uterine cavity by controlling the amount of fluid (by means of Hysteroflator). By using the loop as the curettage, trophoblastic tissues were removed by gentle motions without current application. We did not use misoprostol or prostaglandins for cervical preparing. Seven days after the procedure, betahCG was evaluated again.

Primary outcome was evaluation of changing in beta-hCG levels after 1 and 7 days (a 10 log reduction regarding the primary levels). Secondary outcome was evaluation of the endometrial cavity for PGTD 7 days after the procedure by transvaginal ultrasound (Siemens probe, by a same radiologist who performed the first TVS). If beta-hCG had a plateaued or non-decreasing trend (<a 10 log reduction), the patient was candidate for chemotherapy. All the participants followed by measuring beta-hCG levels for 6 months, until the levels would be undetectable.

\section{Ethical statements}

All participants sign the written informed consent. The oncologist explained the potential pros and cons of hysteroscopic resection and the advantages and disadvantages of chemotherapy as an alternative treatment. Participants could choose the treatment modality on that appointment or informed the authors at separate session to accept or reject the trial. The study had been approved by TUMS ethics committee (Reference Number: 27546-30-03-94). It was registered in Iranian Registry of Clinical Trials (IRCT), (Reference Number: IRCT201601222576N11, 8/2016). This trial was conducted according to the principles of the Helsinki Declaration.

\section{Inclusion and exclusion criteria}

Inclusion criteria were the history of hydatidiform mole that beta-hCG titer in the follow-up did not reach negative value after uterine evacuation or the detecting PGTD in uterus by ultrasound. They were all eager to participate in the study. Exclusion criteria were patients who preferred chemotherapy instead of hysteroscopy.

\section{Statistical analysis}

Data analysis was conducted by means of STATA version 14. (StataCorp, College Station, TX, USA). The Mann Whitney $U$ test was used for data analysis. $P$ value less than 0.05 considered significant. A CONSORT guideline was used for reporting the results.

\section{Results}

Totally 287 participants with confirmed pathology of molar pregnancy underwent uterine evacuation. In the follow-up, 41 cases with persisted PGD criteria enrolled. After a complete evaluation and a consultation by an oncologist, 7 women withdrew from the study and prefer to receive conventional chemotherapy as a treatment of choice. All participants were assessed using FIGO criteria for diagnosis of post-molar gestational trophoblastic neoplasia (14). Low risk GTN was defined as non-metastatic 
(stage 1) or metastatic GTN whose prognostic score was less than 7. We included all non-metastatic, low risk GTNs (30 cases) for hysteroscopy (Figure 1). The mean \pm SD age of patients was $31.4 \pm 4.6$ years. The mean $\pm S D$ of baseline and discharge beta-hCG was $8168.4 \pm 1758$ and $2648.8 \pm 5888(p=0.06)$ respectively.

In follow-up by ultrasound 7 days after hysteroscopy, no PGTD in uterus was reported in all cases. Two patients received chemotherapy after hysteroscopy due to increased beta-hCG level.

There was no significant difference between two groups who received or not received chemotherapy regarding age, gravidity, baseline and discharge beta-hCG Titer (Table 1).

No adverse events during the hysteroscopy such as uterine perforation, volume overload, hemorrhage, or pulmonary emboli happened. In 6 months follow-up after the procedure, all 28 patients were checked routinely and none of them required salvage chemotherapy during this period.

\section{Discussion}

We assess the role of hysteroscopic resection without electrical current in PGTD treatment. The results of this study showed that hysteroscopic resection could treat (28) $93.3 \%$ of the patients with PGTD and only 2 cases $(6.6 \%)$ needed further chemotherapy. Rein et al evaluated 95 patients with non-molar retained products of conception who underwent hysteroscopy or D\&C. However, we included PGTD and resection without electrical current was performed (12).

The standard treatment for $\mathrm{HM}$ is uterine evacuation, but repeated procedure may be associated with complications such as uterine perforation, and intrauterine synechiae (15), however in present study due to direct visualization of uterine cavity and border of the trophoblastic tissues, there was no perforation.

The prevalence of intrauterine adhesions after delayed removal of placental tissues and repeated D\&C reported up to $40 \%$ (16). Faivre et al also reported lower rate of adhesion after hysteroscopic resection of residual trophoblastic tissues. They used hysteroscopy for removing residual trophoblastic tissue in 50 patients and reported $100 \%$ complete evacuation of the uterus with minimal complication (17). In other study, patients with low-risk gestational trophoblastic neoplasia underwent repeat uterine evacuation and revealed that $83 \%$ did not require further chemotherapy (18). In current study only two cases (6.6\%) needed further chemotherapy. Indeed, second curettage for low risk non metastatic GTN was performed in 64 patients and reported $40 \%$ cure after second curettage. They also observed surgical failure in $59 \%$ (19). In contrast, in the present study no complication was reported.

In the other study by Pezeshki et al. 544 patients underwent second uterine evacuation for low risk GTN, while $67 \%$ cured but third evacuation was performed in 28 patients and at last, $27 \%$ of cases needed chemotherapy (20).

This study had some limitation. First the study had selection bias because it is a single arm study. Due to ethical concern, we could not include a control group with second curettage because it was assumed that D\&C may increase risk of perforation or adhesion or incomplete evacuation due to blind nature of D\&C. 
Second, the sample size was limited. So, larger multicentric, randomized studies with follow up protocol and control group (may be D\&C or chemotherapy group) is crucial. The strengths of the study were the novelty in using resection without electrical current and the 6-month follow-up period.

\section{Conclusion}

Hysteroscopy is a safe method that under direct visualization, complete evacuation of uterine cavity and removal of all PGTD is possible, therefore it may be helpful in the management of PGTD and avoid the need for chemotherapy in selected patients.

\section{Abbreviations}

\section{GTN}

gestational trophoblastic neoplasia

PGTD

persistent gestational trophoblastic disease

D\&C

dilatation \& curettage

$\mathrm{HM}$

hydatidiform mole

GTN

gestational trophoblastic neoplasia

SD

standard deviation

$\mathrm{CBC}$

complete blood count CT:computed tomography

MRI

magnetic resonance imaging

ECL

Electrochemiluminescence

IRCT

Iranian Registry of Clinical Trials

TVS

transvaginal sonography

FIGO

International Federation of Gynecologists and Obstetricians

\section{Declarations}

\section{Ethics approval and consent to participate}


All the participants in the study were asked to fill informed consent for participation in the study. The study was approved by the ethical committee of Tehran University of Medical Sciences.

\section{Consent for publication}

All the participants were informed about publication of data anonymously, and they signed the informed consent.

\section{Availability of data and material}

All data analyzed during this study are included in this published article as a supplementary file.

\section{Competing of interests}

The authors declare that they have no competing interests.

\section{Funding}

There is no funding for this study

\section{Acknowledgements}

The authors would like to thanks the staff of perinatology clinic of Woman Hospital for their support and help our patients to fill the questionnaires.

\section{References}

1. Candelier JJ. The hydatidiform mole. Cell Adh Migr. 2016 Mar 3;10(1-2):226-35

2. Candelier JJ (2015 Oct) [Complete hydatidiform mole]. Med Sci (Paris) 31(10):861-868

3. Vassilakos P, Riotton G, Kajii T. Hydatidiform mole: two entities. A morphologic and cytogenetic study with some clinical consideration. Am J Obstet Gynecol. 1977 Jan 15;127(2):167-70

4. Altieri A, Franceschi S, Ferlay J, Smith J, La Vecchia C (2003 Nov) Epidemiology and aetiology of gestational trophoblastic diseases. Lancet Oncol 4(11):670-678

5. Lurain JR (2010 Dec) Gestational trophoblastic disease I: epidemiology, pathology, clinical presentation and diagnosis of gestational trophoblastic disease, and management of hydatidiform mole. Am J Obstet Gynecol 203(6):531-539

6. Sand PK, Lurain JR, Brewer JI (1984 Feb) Repeat gestational trophoblastic disease. Obstet Gynecol 63(2):140-144

7. Akinlaja O, McKendrick R, Mashak Z, Nokkaew M (2016) Incidental Finding of Persistent Hydatidiform Mole in an Adolescent on Depo-Provera. Case Rep Obstet Gynecol 2016:6075049

8. Sasaki S (2003) Clinical presentation and management of molar pregnancy. Best Practice Research Clinical Obstetrics Gynaecology 17(6):885-892 
9. Padron L, Rezende Filho J, Amim Junior J, Sun SY, Charry RC, Maesta I et al (2018 Apr) Manual Compared With Electric Vacuum Aspiration for Treatment of Molar Pregnancy. Obstet Gynecol 131(4):652-659

10. Bagshawe K, Dent J, Webb J (1986) Hydatidiform mole in England andWales The Lancet 328(8508):673-677

11. Wolfberg AJ, Berkowitz RS, Goldstein DP, Feltmate C, Lieberman E (2005 Sep) Postevacuation hCG levels and risk of gestational trophoblastic neoplasia in women with complete molar pregnancy. Obstet Gynecol 106(3):548-552

12. Rein DT, Schmidt T, Hess AP, Volkmer A, Schondorf T, Breidenbach M (2011 Nov-Dec) Hysteroscopic management of residual trophoblastic tissue is superior to ultrasound-guided curettage. J Minim Invasive Gynecol 18(6):774-778

13. Lima LLA, Padron L, Camara R, Sun SY, Rezende JF, Braga A (2017 Jan-Feb) The role of surgery in the management of women with gestational trophoblastic disease. Rev Col Bras Cir 44(1):94-101

14. Kohorn El (2001 Jan-Feb) The new FIGO 2000 staging and risk factor scoring system for gestational trophoblastic disease: description and critical assessment. Int J Gynecol Cancer 11(1):73-77

15. Thomson AJA, Jason A, Deans R, Kingston A, Vancaillie (2009) Thierry Gb. The management of intrauterine synechiae. CurrentOpinion in Obstetrics Gynecology 21(4):335-341

16. Westendorp I, Ankum WM, Mol BW, Vonk J (1998) Prevalence of Asherman's syndrome after secondary of placental remnants or a repeat curettage for incomplete abortion. Hum Reprod 13(12):3347-3350

17. Faivre E, Xavier Deffieux C, Mrazguia et al (2009) Hysteroscopic management of residual trophoblastic tissue and reproductive outcome: a pilot stu. Journal of minimally invasivegynecology 16(4):487-490

18. Yarandi F, Jafari F, Shojaei H, Izadi-Mood N (2014) Clinical response to a second uterine curettage in patientswith low-risk gestational trophoblastic disease: a pilot study. J Reprod Med 59(11-12):566570

19. Osborne RJFV, Schink JC et al (2016) Second Curettage for Low-Risk Nonmetastatic Gestational Trophoblastic Neoplasia. Obstet Gynecol 128(3):535-542

20. Pezeshki MHB, Silcocks P, Everard JE, Coleman J, Gillespie AM, Tidy J, Coleman RE (2004) The role of repeat uterine evacuation in the management of persistent gestational trophoblastic disease. Gynecol Oncol 95(3):423-429

\section{Tables}

Table 1: Comparison the demographic and clinical factors between 2 groups who received or not received chemotherapy. 


\begin{tabular}{|llll|}
\hline P value & $\begin{array}{l}\text { Chemotherapy }(-) \\
\mathrm{n}=\mathbf{2 8}\end{array}$ & $\begin{array}{l}\text { Chemotherapy }(+) \\
\mathrm{n}=\mathbf{2}\end{array}$ \\
\hline $\mathbf{0 . 6 0 0}$ & $31.5 \pm 4.7$ & $30 \pm 1.4$ & Age \\
\hline $\mathbf{0 . 6 0 0}$ & $2.4 \pm 1.1$ & $2 \pm 1.4$ & Gravidity \\
\hline $\mathbf{0 . 0 8 0}$ & $6561 \pm 1718$ & $29053 \pm 5448$ & Baseline beta-hCG \\
\hline $\mathbf{0 . 5 0 0}$ & $2436 \pm 604$ & $5195 \pm 331$ & Beta-hCG in day 7 \\
\hline
\end{tabular}

$P$ value $<0.05$ is significant 